\title{
OPTIMIERUNGSAUFGABEN IN UNTERNEHMENSPROJEKTEN
}

\section{Detlev Ruland, Siemens AG, München}

Unternehmensprojekte werden segmentiert in die zwei Klassen:

a) Strategie-Projekte:

Ausrichtung (neuer) Geschäfte, Spezifikation der Strategie und wirtschaftliche Ziele, Ableitung von Maßnahmen

b) Restrukturierungs-Projekte:

Analyse der Ursachen für verlustträchtige Geschäfte, Identifikation von Ansatzpunkten und Spezifikation von Maßnahmen, Umsetzung und Kontrolle der Maßnahmen

In beiden Projektklassen sind zahlreiche Problemstellungen aus dem Optimierungsbereich zu lösen, die größtenteils mit traditionellen Verfahren und Algorithmen angegangen werden können. Im Rahmen des Vortrags wird daher schwerpunktmäßig auf die Problemstellungen und weniger auf die Lösungsansätze eingegangen.

Im folgenden sind einige typische Analysen diskutiert, die in Unternehmensprojekten zu Anwendungen kommen. Anhand dieser Beispiele werden die dort auftretenden Optimierungsaufgaben exemplarisch diskutiert:

1. Analyse von Losgrößen

2. Analyse der Leistungstiefe

3. Analyse von Fertigungsstandortkonzepten

4. Analyse von Vertrieb-Länderportfolios

5. Analyse von Komplexitätskosten

6. Analyse von FuE-Kosten/FuE-Programm

7. Analyse zum Target Costing 